\title{
Enhancing elastic stress relaxation in SiGe/Si heterostructures by Si pillar necking
}

F. Isa', M. Salvalaglio, Y. Arroyo Rojas Dasilva, A. Jung, G. Isella, R. Erni, B. Timotijevic, P. Niedermann, P. Gröning, F. Montalenti, and $H$. von Känel

Citation: Appl. Phys. Lett. 109, 182112 (2016); doi: 10.1063/1.4966948

View online: http://dx.doi.org/10.1063/1.4966948

View Table of Contents: http://aip.scitation.org/toc/apl/109/18

Published by the American Institute of Physics

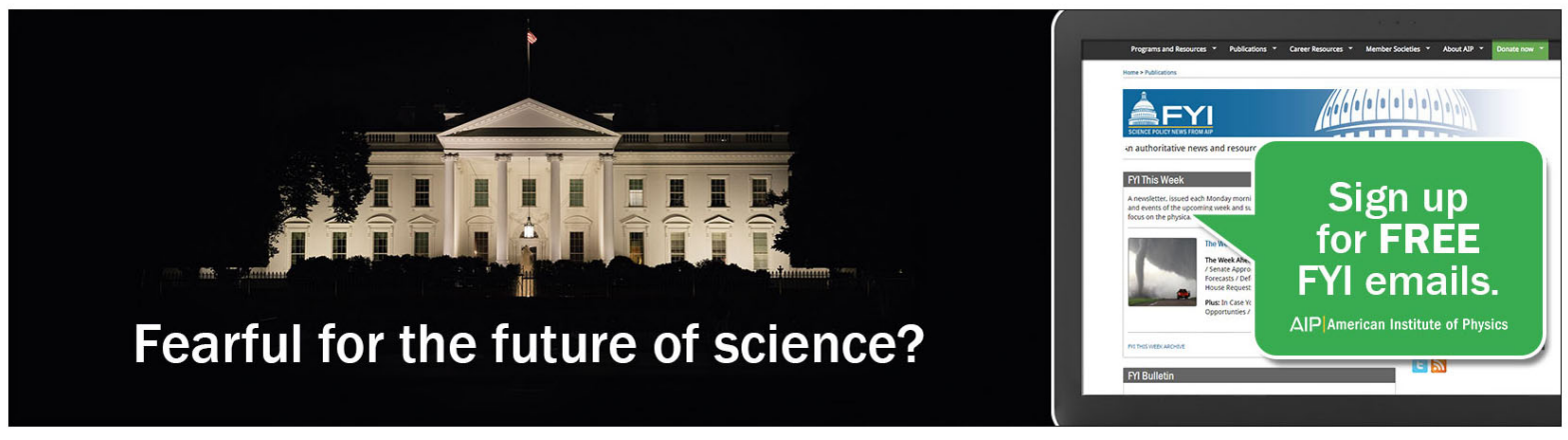




\title{
Enhancing elastic stress relaxation in SiGe/Si heterostructures by Si pillar necking
}

\author{
F. Isa, ${ }^{1,2, a)}$ M. Salvalaglio, ${ }^{3,4}$ Y. Arroyo Rojas Dasilva, ${ }^{2}$ A. Jung, ${ }^{1,2}$ G. Isella, ${ }^{5}$ R. Erni, ${ }^{2}$ \\ B. Timotijevic, ${ }^{6}$ P. Niedermann, ${ }^{6}$ P. Gröning, ${ }^{7}$ F. Montalenti, ${ }^{3}$ and H. von Känel ${ }^{1,2}$ \\ ${ }^{1}$ Laboratory for Solid State Physics, ETH Zürich, Otto-Stern-Weg 1, Zürich CH-8093, Switzerland \\ ${ }^{2}$ Electron Microscopy Center Empa, Swiss Federal Laboratories for Materials Science and Technology, \\ Überlandstrasse 129, Dübendorf CH-8600, Switzerland \\ ${ }^{3}$ L-NESS and Department of Materials Science, Università di Milano-Bicocca, Via Cozzi 55, \\ Milano I-20125, Italy \\ ${ }^{4}$ Institute for Scientific Computing, Technische Universität Dresden, Willers-Bau B - Zellescher Weg 12-14, \\ Dresden D-01062, Germany \\ ${ }^{5}$ L-NESS and Department of Physics, Politecnico di Milano and IFN-CNR, Via Anzani 42, Como I-22100, Italy \\ ${ }^{6}$ CSEM, Rue Jaquet-Droz 1, Neuchâtel CH-2002, Switzerland \\ ${ }^{7}$ Advanced Materials and Surfaces Department Empa, Swiss Federal Laboratories for Materials Science \\ and Technology, Überlandstrasse 129, Dübendorf CH-8600, Switzerland
}

(Received 6 June 2016; accepted 21 October 2016; published online 3 November 2016)

\begin{abstract}
We demonstrate that the elastic stress relaxation mechanism in micrometre-sized, highly mismatched heterostructures may be enhanced by employing patterned substrates in the form of necked pillars, resulting in a significant reduction of the dislocation density. Compositionally graded $\mathrm{Si}_{1-x} \mathrm{Ge}_{x}$ crystals were grown by low energy plasma enhanced chemical vapour deposition, resulting in tens of micrometres tall, three-dimensional heterostructures. The patterned $\mathrm{Si}(001)$ substrates consist of micrometre-sized Si pillars either with the vertical $\{110\}$ or isotropically under-etched sidewalls resulting in narrow necks. The structural properties of these heterostructures were investigated by defect etching and transmission electron microscopy. We show that the dislocation density, and hence the competition between elastic and plastic stress relaxation, is highly influenced by the shape of the substrate necks and their proximity to the mismatched epitaxial material. The SiGe dislocation density increases monotonically with the crystal width but is significantly reduced by the substrate under-etching. The drop in dislocation density is interpreted as a direct effect of the enhanced compliance of the under-etched Si pillars, as confirmed by the three-dimensional finite element method simulations of the elastic energy distribution. Published by AIP Publishing.

[http://dx.doi.org/10.1063/1.4966948]
\end{abstract}

The epitaxial integration of dissimilar semiconductors plays a fundamental role in the "more than Moore" paradigm in which materials with superior electro-optical properties are combined with silicon., ${ }^{1,2}$ Thin compressively strained epitaxial layers (e.g., $\mathrm{SiGe} / \mathrm{Si}$ ) may relax by surface undulation, ${ }^{3}$ by island and cluster formation ${ }^{4}$ and eventually by extended defects such as misfit dislocations (MDs) and threading dislocations (TDs) for layer thicknesses exceeding a certain critical value. Dislocations are introduced at or near the heterointerface to accommodate plastically the lattice mismatch ${ }^{5}$ and negatively affect the electro-optical properties of devices. ${ }^{6,7}$

The TD density may be drastically reduced or even eliminated by thermal treatments, ${ }^{8}$ by compositionally graded buffer layers, ${ }^{9}$ by patterned substrates and aspect ratio trapping, ${ }^{10}$ by three-dimensional (3D) heteroepitaxy ${ }^{11}$ or by pendeoepitaxy. ${ }^{12}$ Conversely, MDs are equilibrium defects directly related to the epilayer/substrate lattice mismatch. The only way to grow thick, relaxed crystals with a lower density of MDs is to engineer the mechanical properties of the substrate by enhancing its compliance. ${ }^{13,14}$

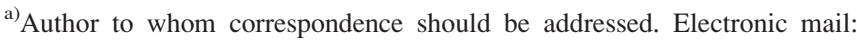
isaf@phys.ethz.ch
}

Recently, we have demonstrated that highly mismatched and coherent (i.e., MD-free) $\mathrm{SiGe} / \mathrm{Si}$ heterostructures may be obtained at the micrometre-scale by combining shallow grading of the $\mathrm{Ge}$ content and growth at a finite lateral size. ${ }^{15}$ The maximum width of the coherent heterostructures was limited to about $6 \mu \mathrm{m}$, while for larger sizes the plastic stress relaxation mechanism became effective.

Here, we demonstrate that the compliance of patterned Si substrates may be significantly enhanced by employing the under-etched pillar structures giving access to wider heterostructures with low dislocation densities both at the $\mathrm{SiGe/}$ Si heterointerface and within the SiGe crystals.

The experimental results, based on the evaluation of the dislocation density in the SiGe crystals, are supported by the simulations of residual misfit stress by the finite element method (FEM).

The SiGe crystals were epitaxially grown on the 4-in. n-type (3-7 $\Omega \mathrm{cm}) \mathrm{Si}(001)$ substrates patterned by two different approaches. The first one, as illustrated in Figure 1(a), consists of the standard optical lithography followed by the Bosch process ${ }^{16}$ and leads to the regular arrays of $8 \mu \mathrm{m}$ tall square Si pillars with vertical $\{110\}$ sidewalls. ${ }^{17}$ The width of the Si pillars ranges from 2 to $50 \mu \mathrm{m}$ and they are separated by 3,4 or $5 \mu \mathrm{m}$ wide gaps. The second approach, as shown in Figure 1(b), is accomplished in two steps. In the 

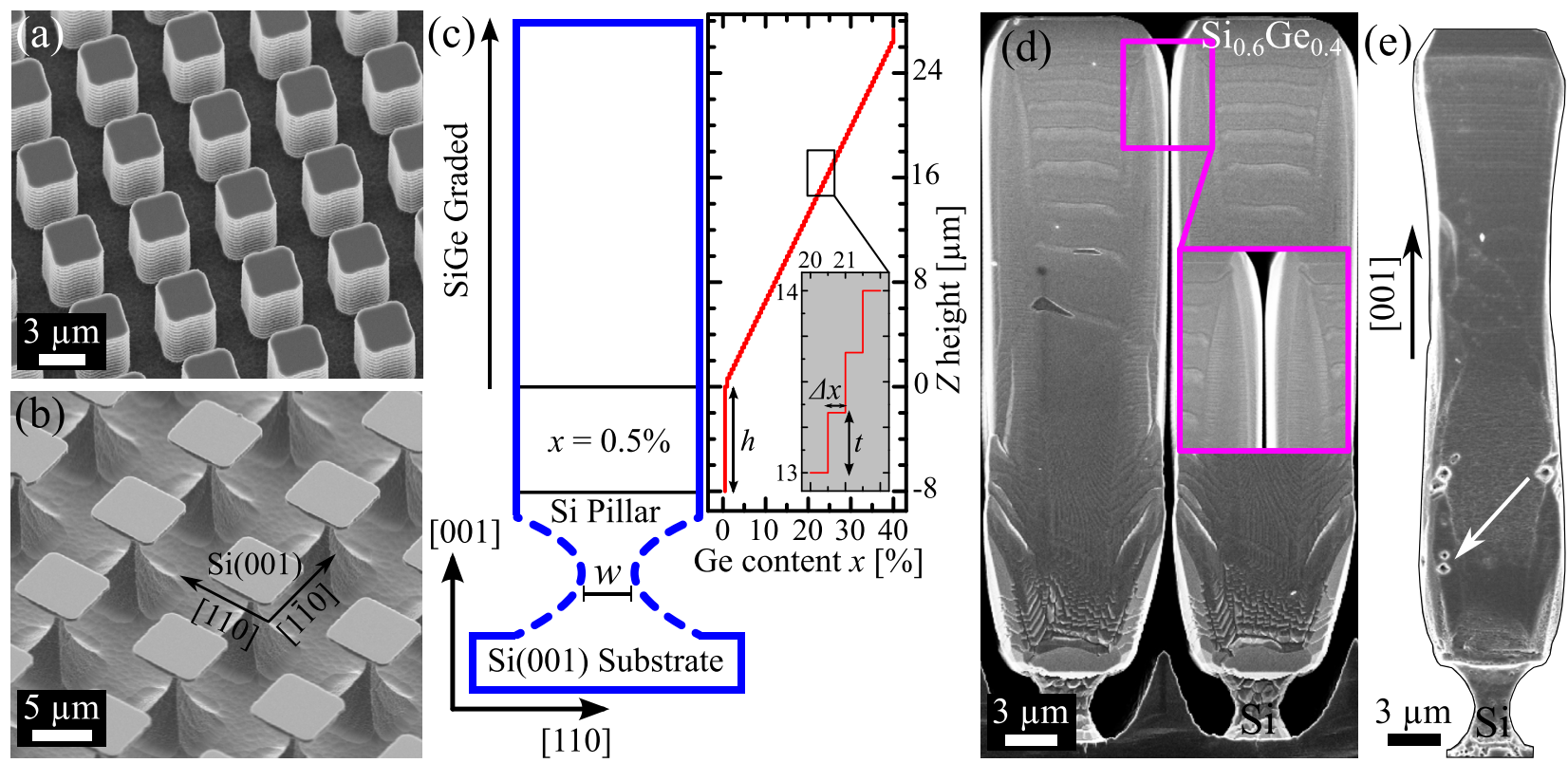

FIG. 1. (a) and (b) SEM images of vertical and under-etched Si pillars, respectively. In (b), the width of the Si pillar necks is $\sim 1 \mu \mathrm{m}$ and the top square membrane is $1 \mu \mathrm{m}$ thick. (c) Sample schematic consisting of the Si pillar (under-etched, dashed line) followed by a spacer layer of thickness $h$ and $\mathrm{Ge}$ content $0.5 \%$, and finally the compositionally graded part $\left(\mathrm{GR}=1.5 \% \mu \mathrm{m}^{-1}\right)$ with a $1 \mu \mathrm{m}$ thick cap layer at $x_{f}=0.4$. Each layer in the graded part has a thickness $t \sim 333 \mathrm{~nm}$ and a Ge content increased by $\Delta x=0.5 \%$ with respect to the previous one. The width of the Si pillar neck is $w$. (d) Cross-sectional SEM image of two graded SiGe crystals $(h=50 \mathrm{~nm})$ deposited on under-etched, $7 \mu \mathrm{m}$ wide Si pillars. The magenta inset shows a magnification of the nanometre wide gaps between the crystals. (e) Same as (d) but after defect etching. One etch pit indicating an emerging dislocation is marked by the white arrow.

first step, vertical $0.6-1 \mu \mathrm{m}$ tall mesas are defined by the Bosch process. In the second step, necks are formed by $\sim 3 \mu \mathrm{m}$ isotropic dry under-etching by reactive ion etching with an inductively coupled plasma and $\mathrm{SF}_{6}$ and $\mathrm{O}_{2}$ gas under low bias conditions. The minimum width of the original Si pillars is $7 \mu \mathrm{m}$ resulting in $1 \mu \mathrm{m}$ wide necks in the under-etched region (indicated by $w$ in Figure 1(c)). Smaller Si pillars are not possible to process. Larger Si pillars have comparable under-etching and wider necks.

The epitaxial growth was performed by low energy plasma enhanced chemical vapour deposition (LEPECVD). ${ }^{18}$ The sample schematic is displayed in Figure 1(c). It consists first of a low Ge content $(x=0.5 \%)$ spacer layer of either thickness $h=50 \mathrm{~nm}$ or $8 \mu \mathrm{m}$ and deposited at $750^{\circ} \mathrm{C}$ and $5.9 \mathrm{~nm} / \mathrm{s}$. Thanks to the very low Ge content, this spacer layer is fully coherent for all the Si pillar widths, ${ }^{19}$ and its thickness $h$ determines the distance between the mismatched epitaxial material and the Si pillar necks. It is followed by a compositionally graded $\mathrm{Si}_{1-x} \mathrm{Ge}_{x}$ alloy where $x$ is stepwise increased (see Figure 1(c)) at a grading rate (GR) of $1.5 \% \mu \mathrm{m}^{-1}$ up to the final $\mathrm{Ge}$ content $x_{f}=0.4$. The growth temperature is kept at $750^{\circ} \mathrm{C}$ for $x<0.1$ and then linearly decreased to $590^{\circ} \mathrm{C}$ reached at $x_{f}=0.4$. The growth rate varies between 5.9 and $10 \mathrm{~nm} / \mathrm{s}$ in the Ge compositional range $0.5 \%<x$ $<0.4$. Each step has a thickness of $t \sim 333 \mathrm{~nm}$ and the $\mathrm{Ge}$ content augmented by $\Delta x=0.5 \%$. Finally, a $1 \mu \mathrm{m}$ thick cap layer $\left(x_{f}=0.4\right)$ is deposited at $590^{\circ} \mathrm{C}$. As demonstrated in Figure 1(d), SiGe crystals of tens of micrometres tall are obtained, which are separated by nanometre wide gaps ${ }^{11}$ (see magenta inset). They have $\{110\}$ sidewalls and $\{111\},\{113\}$ and (001) top facets.

In order to evaluate if the $\mathrm{SiGe} / \mathrm{Si}$ lattice misfit is elastically or plastically relaxed by means of dislocations, a transmission electron microscopy (TEM) analysis and defect etching (solution consisting of: $\mathrm{CrO}_{3}, 10.8 \mathrm{~g}+\mathrm{HF} 40 \%$, $110 \mathrm{ml}+$ deionized water, $113 \mathrm{ml}$ at $0{ }^{\circ} \mathrm{C}$ ) followed by etch pit counting by scanning electron microscopy (SEM) were carried out.

The etch pit counting was performed in cross-section on the $\{110\}$ crystal sidewalls on an equivalent area ranging from $7 \times 10^{3}$ to $2 \times 10^{4} \mu \mathrm{m}^{2}$, which corresponds to tens of different crystals, ensuring reliable statistics. The investigated area consists of the crystal region extending from the Si pillar/spacer layer heterointerface to the $x_{f}=0.4$ cap layer. In this way, both dislocations close to the heterointerface as well as those within the graded alloy are taken into account. This approach significantly differs from the typical one where only TDs reaching the top surface may be detected. ${ }^{20}$ The dislocation density is evaluated by counting the number of etch pits on the $\{110\}$ sidewalls of each crystal and calculating the average value and related standard deviation. Both MDs and TDs, which can be distinguished by the different etch pit shape, are taken into account to calculate the dislocation density. The total (MDs + TDs) dislocation density is more representative to estimate the competition between the elastic/plastic strain relaxation mechanism since only the defects appearing on the $\{110\}$ sidewalls are counted, and MDs may interact and block each other within the SiGe crystals. The etch pit shape depends on the inclination of the dislocation line $l$ with respect to the $\{110\}$ sidewall, where they are observed. The etch pit shape of MDs is 4-fold symmetric ( $l$ along $\langle 110\rangle)$, while asymmetric and elongated for TDs ( $l$ in $\{111\}$ planes); for further details, see Refs. 19 and 21. Additionally, the probability of dislocation-free crystals is calculated by counting the number of crystals without etch pits divided by the total number of investigated ones. 
Figure 1(e) shows an SEM image of a graded SiGe crystal after defect etching. Emerging dislocations are visible as pits on the $\{110\}$ sidewall, one of which is indicated by the white arrow.

Figure 2(a) shows the dislocation density of SiGe crystals as a function of their width, grown on vertical Si pillars with $h=8 \mu \mathrm{m}$ (black spheres) and under-etched Si pillars with $h=8 \mu \mathrm{m}$ (red triangles) and $h=50 \mathrm{~nm}$ (green triangles). For the narrowest heterostructures $6 \mu \mathrm{m}$ in width on vertical Si pillars (indicated by the black arrow), elastic stress relaxation dominates; indeed, no dislocations can be seen in accordance with Refs. 15, 19, and 22. High resolution X-ray diffraction and micro-Raman measurements (not shown here) indicate that the SiGe crystals are fully strain relaxed (within the experimental accuracy) for every crystal width (for further details, see Refs. 15 and 23).

For the SiGe crystal widths exceeding $6 \mu \mathrm{m}$, we find a monotonically increasing dislocation density for all pillar shapes and spacer thicknesses $h$.

Moreover, if $h=8 \mu \mathrm{m}$, we find comparable dislocation densities for SiGe crystals on both the vertical and underetched Si pillars. This finding indicates that the elastic relaxation mechanism is barely affected by the Si pillar shape when the mismatched SiGe material is not in proximity to the pillar necks.

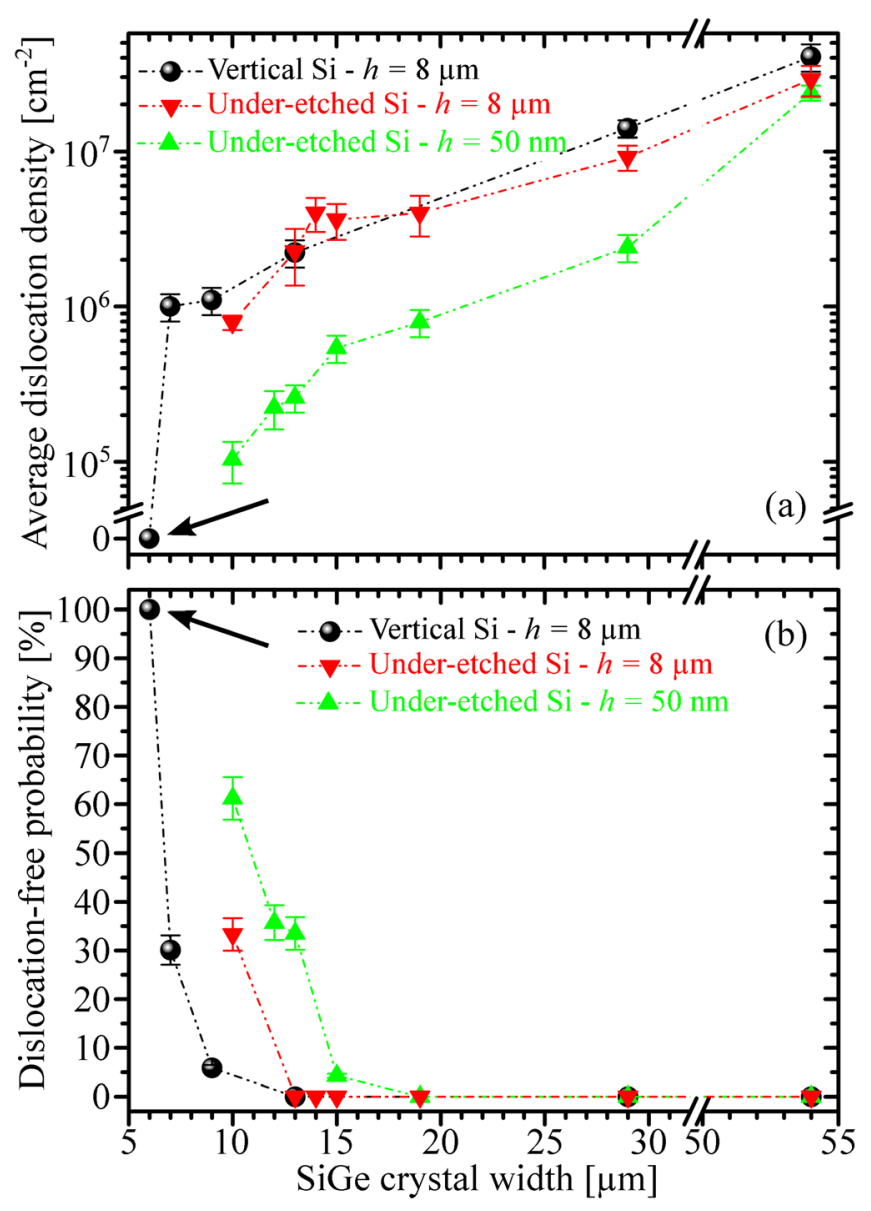

FIG. 2. (a) Average dislocation density (MDs $+\mathrm{TDs}$ ) in SiGe crystals with different widths deposited on vertical $\mathrm{Si}$ pillars with $h=8 \mu \mathrm{m}$ (black spheres) and under-etched Si pillars with $h=8 \mu \mathrm{m}$ (red triangles) and $h=50 \mathrm{~nm}$ (green triangles). (b) Probability of having dislocation-free SiGe crystals as a function of their width.
For thin $(h=50 \mathrm{~nm})$ spacer layers and crystal widths $\leq 29 \mu \mathrm{m}$, pillar necking significantly affects the dislocation density and therefore the stress relaxation mechanism. Conversely, for wider SiGe crystals, the dislocation densities appear to converge independently of $h$ and Si pillar shape. For example, the dislocation density is $\sim 4 \times 10^{7} \mathrm{~cm}^{-2}$ at a crystal width of $54 \mu \mathrm{m}$ in all the samples.

These results demonstrate that suitable Si pillar necking increases the compliance of the patterned substrate. In other words, the onset of dislocation nucleation is delayed in favour of elastic stress relaxation. This effect becomes negligible for wide structures since in that case the width of the necks is much larger than the amount of under-etching $(\sim 3 \mu \mathrm{m})$.

Figure 2(b) shows the probability of finding a dislocation-free SiGe crystal as a function of its width. As mentioned before, $6 \mu \mathrm{m}$ wide SiGe crystals on vertical Si pillars (black arrow) are 100\% (within the experimental statistics) dislocation-free. By increasing the crystal width to 7 and $9 \mu \mathrm{m}$, the dislocation density increases abruptly from zero to $1.0 \times 10^{6} \mathrm{~cm}^{-2}$ and $1.1 \times 10^{6} \mathrm{~cm}^{-2}$, but still the probability to find a dislocation-free crystal is $30 \%$ and $6 \%$, respectively. The probability of finding dislocation-free crystals drops to zero for widths $\geq 9 \mu \mathrm{m}$. The same result is obtained for the SiGe crystals on under-etched Si pillars in case of $h=8 \mu \mathrm{m}$.

Conversely, the critical width at which the probability for dislocation-free crystals drops to zero is $19 \mu \mathrm{m}$ for SiGe crystals deposited on under-etched Si pillars provided that the spacer layer is $h=50 \mathrm{~nm}$ thin (green triangles). These findings again show that the mechanical behaviour of the two heterostructures with $h=8 \mu \mathrm{m}$ is very similar while under-etched Si pillars with $h=50 \mathrm{~nm}$ are more effective in elastic stress relaxation.

Dislocations in SiGe crystals were also investigated by TEM. Even though the TEM statistics is inferior to that provided by the etch pit counting, Figure 3(a) shows (for the same crystal width) a larger density of dislocations in the SiGe crystals with $h=8 \mu \mathrm{m}$ than Figure 3(b), in which a crystal with $h=50 \mathrm{~nm}$ is analysed. The Burgers vector $b$ of the dislocations was identified by using the invisibility criterion $(g \cdot b=0)$ and dark-field two beams conditions, with $g=(004)$, ( $2 \overline{2} 0),(20 \overline{2}),(202),(1 \overline{1} 1),(1 \overline{1} \overline{1})$ scattering planes. The analysis indicates that dislocations have the Burgers vector of $a / 2[011], a / 2[10 \overline{1}], a / 2[101]$ or $a / 2[01 \overline{1}]$. The dislocation line $l$ was obtained using the trace analysis method described in Ref. 24, and the vast majority of dislocations have $l=[110]$. These results imply that the dislocations are $60^{\circ}$ (angle between $b$ and $l$ ) and have $\{111\}$ glide planes. The dislocations are arranged in arrays due to their gliding and nucleation mechanism on the same $\{111\}$ plane. This behavior is typical for the heterogeneous dislocation nucleation process of low misfit systems and shallow grading rate alloys. ${ }^{25,26}$

In Figures 3(a) and 3(b), the layered contrast is due to the stepwise change of the Ge content and it indicates the crystal shape evolution during the growth.

The mechanical properties of the $\mathrm{SiGe} / \mathrm{Si}$ crystals were also investigated by the 3D FEM simulations based on the linear elasticity theory. We determined the elastic field by modeling the $\mathrm{SiGe/Si}$ structure, as sketched in Figure 4(a), and solving the mechanical equilibrium equation. The elastic energy density is then computed as $\rho_{e l}=\sum_{i j} \sigma_{i j} \epsilon_{i j}$, where $\epsilon$ 

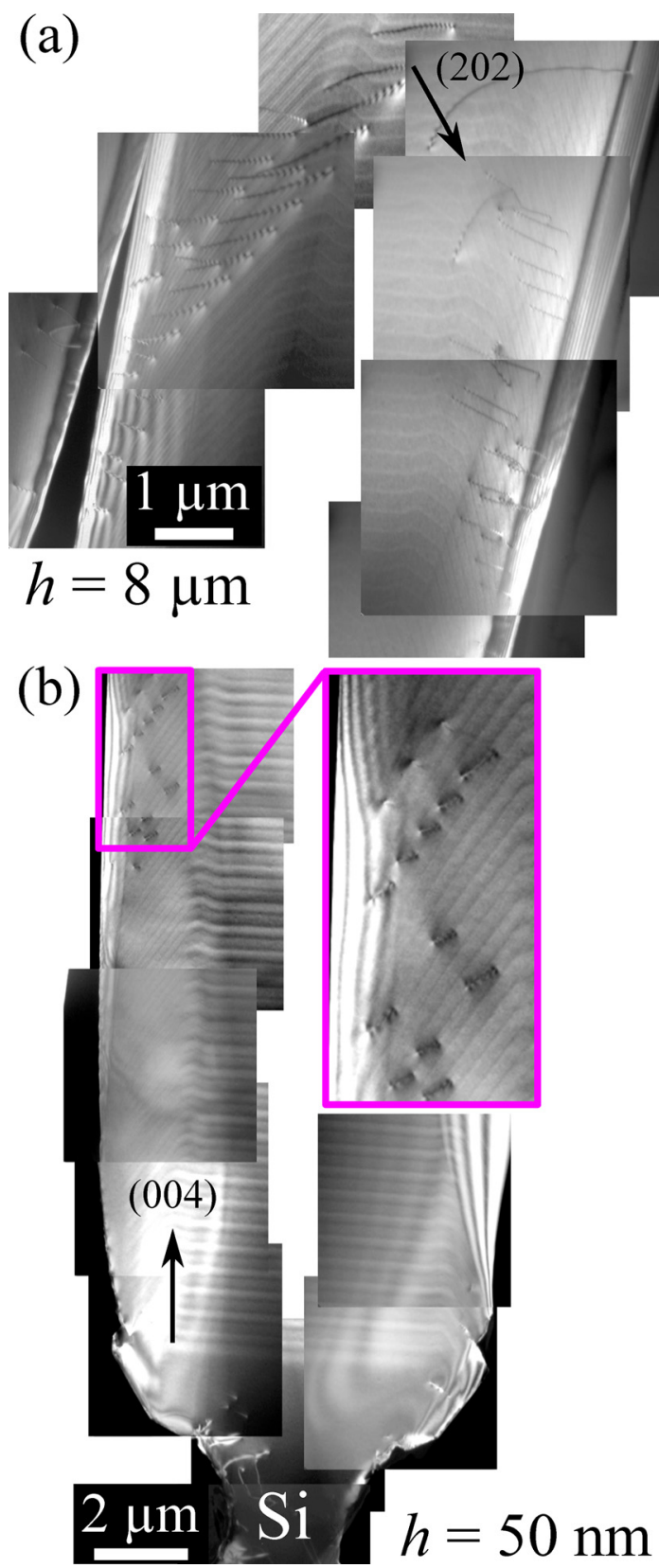

FIG. 3. (a) and (b) Dark-field TEM images under the (202) and (004) diffraction conditions of a SiGe crystal $11 \mu \mathrm{m}$ in width on an under-etched Si pillar with thick $(h=8 \mu \mathrm{m})$ and thin $(h=50 \mathrm{~nm})$ spacer layers, respectively. In (b), the magenta inset shows a magnification of the dislocated area.

is the strain tensor, $\boldsymbol{\sigma}=\boldsymbol{C}: \boldsymbol{\epsilon}$ the stress tensor, and $\boldsymbol{C}$ the stiffness tensor (for further details about the calculations, see Ref. 22). Starting from the experimental (SEM) crystal profile, a simplified cylindrical 3D geometry is obtained by a rotation around the $z$-[001] axis. The distribution of the Ge content (see Figure 4(b)) along $z$ is approximated by linear grading at a GR of $1.5 \% \mu \mathrm{m}^{-1}$ starting from the top $(z=0)$ of $x=0.5 \%$ spacer layer of thickness $h$.

We limited the calculations to $z<8 \mu \mathrm{m}(x<0.125)$ since the effect of further material deposition on the elastic relaxation process is negligible. Indeed, the defect etching experiments indicate that the vast majority of the dislocations $(89 \%)$ are confined in the region with a Ge content $x<0.125$.

The calculated elastic energy densities $\rho_{\mathrm{el}}$ for a $\mathrm{SiGe}$ crystal on an under-etched and on a vertical $\mathrm{Si}$ pillar

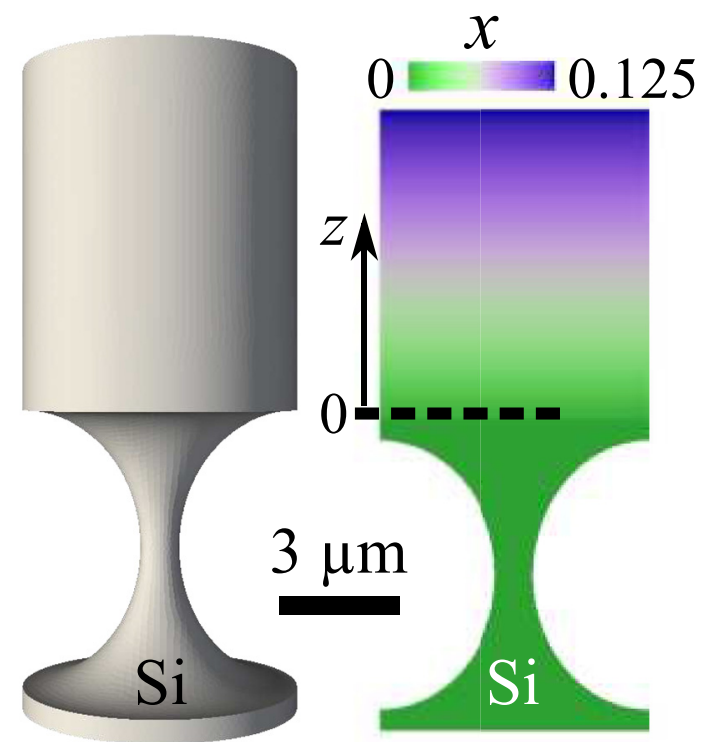

(b)

(a)

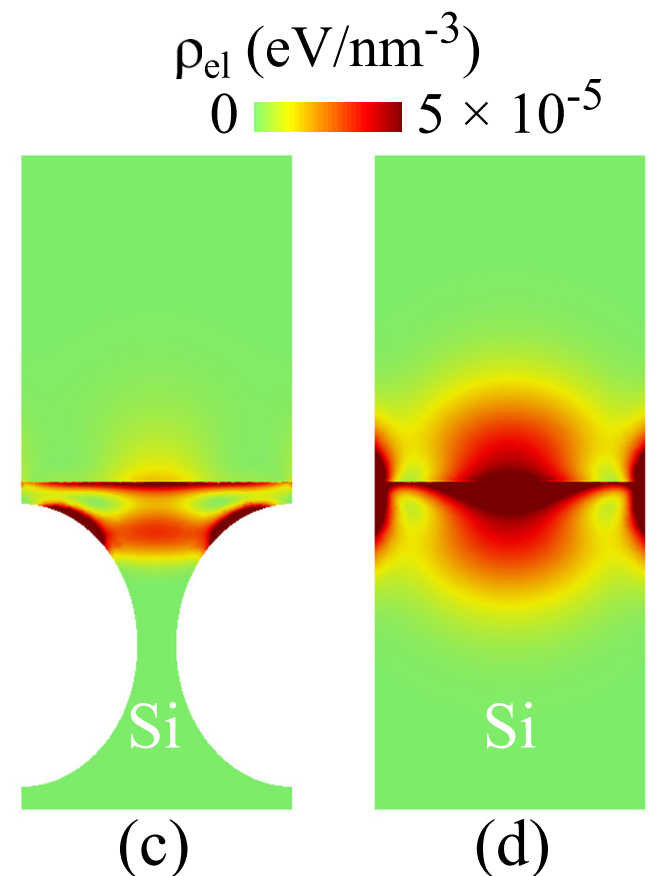

FIG. 4. (a) 3D geometry with cylindrical symmetry of the SiGe/Si structure with under-etched Si pillar and $h=50 \mathrm{~nm}$. (b) Color scale of the Ge content $x$ as a function of $z$. The value $z=0$ corresponds to the top of the $x=0.5 \%$ spacer layer $(h=50 \mathrm{~nm})$. (c) and (d) Elastic energy density $\rho_{\mathrm{el}}$ obtained from the FEM simulations for a SiGe crystal $(h=50 \mathrm{~nm})$ on an under-etched and on a vertical Si pillar, respectively.

( $h=50 \mathrm{~nm}$ ) are shown in Figures 4(c) and 4(d), respectively. By comparing the $\rho_{\mathrm{el}}$ maps in Figures $4(\mathrm{c})$ and 4(d), it can be easily seen that the neck in the Si pillar significantly reduces the spatial extent of the elastic energy such that the latter is much more concentrated close to the interface. The compliance of the substrate is thus enhanced, which allows for more efficient elastic relaxation of the misfit stress and thereby reduces the tendency for dislocation nucleation.

Figure 5 shows $\rho_{\mathrm{el}}$ along $z$ in the center of a SiGe crystal on an under-etched Si pillar for a thin, $h=50 \mathrm{~nm}$ (green solid line), and a thick, $h=8 \mu \mathrm{m}$, spacer layer (red dotted line). For comparison, the corresponding $\rho_{\mathrm{el}}$ for a SiGe crystal on 


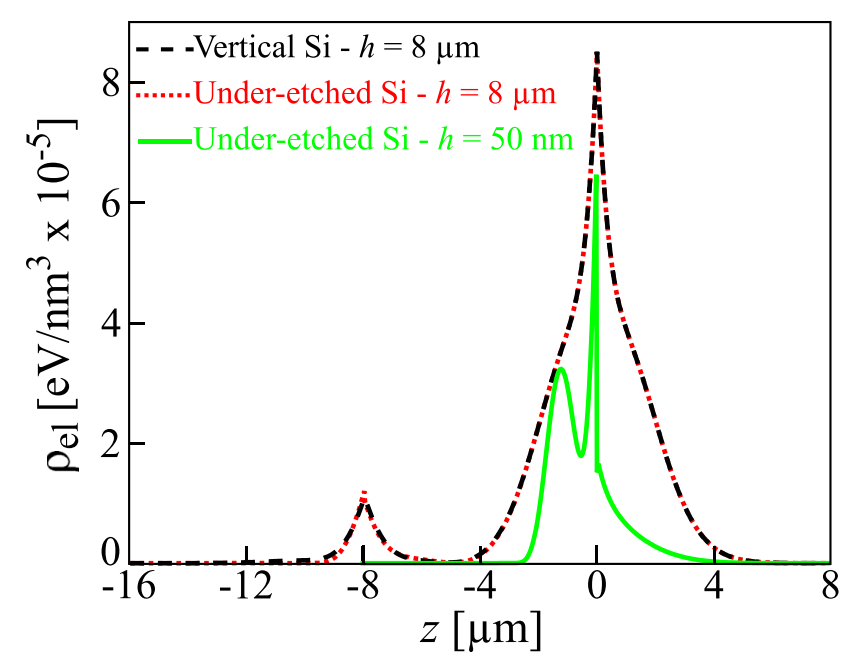

FIG. 5. Elastic energy density $\rho_{\mathrm{el}}$ along the $z$-axis in the center of a SiGe crystal on a vertical Si pillar with $h=8 \mu \mathrm{m}$ (black dashed line) and on an under-etched Si pillar with $h=8 \mu \mathrm{m}$ (red dotted line) and $h=50 \mathrm{~nm}$ (green solid line), respectively. The value $z=0$ corresponds to the top of the $x=0.5 \%$ spacer layer.

a vertical Si pillar with $h=8 \mu \mathrm{m}$ (black dashed line) is also shown. The comparison between the two under-etched structures indicates that elastic stress relaxation is favored by employing a thin spacer layer since in this case the most stressed material is closer to the Si neck. For thick spacers, $\rho_{\mathrm{el}}$ coincides for SiGe crystals on both the under-etched (red dotted line) and vertical Si pillars (black dashed line) apart from a slight difference at $z=-h=-8 \mu \mathrm{m}$. Therefore, the enhancement of the elastic stress relaxation mechanism is not effective if the mismatched epitaxial material is far away from the Si neck.

We verified that any effect of the substrate compliance vanishes for an aspect ratio: $h /$ pillar width $>1$. This is in agreement with the confinement of the misfit stress at the heterointerface in vertical heterostructures. ${ }^{22,27}$

The FEM results thus support the experimental findings reported in Figure 2. Indeed, the dislocation density in the SiGe crystals with $h=8 \mu \mathrm{m}$ is unaffected by the Si pillar shape, i.e., it is similar for the vertical and under-etched pillars. By contrast, if the SiGe crystal is in proximity $(h=50 \mathrm{~nm})$ of the Si substrate neck, a significantly lower dislocation density and an increase in the dislocation-free probability are observed in agreement with the reduction in the elastic energy density (Figures 4 and 5).

In conclusion, we have demonstrated that the dislocation density in micrometer-sized $\mathrm{SiGe} / \mathrm{Si}$ crystals may be significantly reduced by enhancing the substrate compliance via mesa patterning and necking. We have achieved more than $60 \%$ dislocation-free compositionally graded SiGe crystals for the widths of $10 \mu \mathrm{m}$. According to the theoretical calculations, even wider coherent heterostructures may be obtained by further reducing the compositional GR. These heterostructures may be employed to realize vertical transport devices (e.g., thick $\mathrm{SiGe} / \mathrm{Si} \mathrm{X}$-ray absorbers or solar cells) exploiting the absence of dislocations near the heterointerface and within the crystals to reduce the leakage currents. Their width at the micrometer-scale ensures good mechanical stability for device processing.

We acknowledge the Sinergia project NOVIPIX CRSII2_147639 of the Swiss National Science Foundation. We thank Professor B. Batlogg, Professor L. Miglio and Professor Gian-Luca Bona for the fruitful discussions and continuous support. We are grateful to A. Ballabio, the technical staff at the L-NESS laboratory in Como and FIRST clean room at the ETH Zürich.

${ }^{1}$ M. M. Waldrop, Nature 530, 144 (2016).

${ }^{2}$ M. Lundstrom, Science 299, 210 (2003).

${ }^{3}$ T. Walther, C. J. Humphreys, and A. G. Cullis, Appl. Phys. Lett. 71, 809 (1997).

${ }^{4}$ A. J. Steinfort, P. M. L. O. Scholte, A. Ettema, F. Tuinstra, M. Nielsen, E. Landemark, D.-M. Smilgies, R. Feidenhans'1, G. Falkenberg, L. Seehofer, and R. L. Johnson, Phys. Rev. Lett. 77, 2009 (1996).

${ }^{5}$ R. People and J. C. Bean, Appl. Phys. Lett. 47, 322 (1985).

${ }^{6}$ N. G. Weimann, L. F. Eastman, D. Doppalapudi, H. M. Ng, and T. D. Moustakas, J. Appl. Phys. 83, 3656 (1998).

${ }^{7}$ D. Jena, A. C. Gossard, and U. K. Mishra, Appl. Phys. Lett. 76, 1707 (2000).

${ }^{8}$ M. Yamaguchi, A. Yamamoto, M. Tachikawa, Y. Itoh, and M. Sugo, Appl. Phys. Lett. 53, 2293 (1988).

${ }^{9}$ E. A. Fitzgerald, Y.-H. Xie, M. L. Green, D. Brasen, A. R. Kortan, J. Michel, Y.-J. Mii, and B. E. Weir, Appl. Phys. Lett. 59, 811 (1991).

${ }^{10}$ J. Z. Li, J. Bai, J.-S. Park, B. Adekore, K. Fox, M. Carroll, A. Lochtefeld, and Z. Shellenbarger, Appl. Phys. Lett. 91, 21114 (2007).

${ }^{11}$ C. V. Falub, H. von Känel, F. Isa, R. Bergamaschini, A. Marzegalli, D. Chrastina, G. Isella, E. Müller, P. Niedermann, and L. Miglio, Science 335, 1330 (2012).

${ }^{12}$ K. Linthicum, T. Gehrke, D. Thomson, E. Carlson, P. Rajagopal, T. Smith, D. Batchelor, and R. Davis, Appl. Phys. Lett. 75, 196 (1999).

${ }^{13}$ D. Zubia and S. D. Hersee, J. Appl. Phys. 85, 6492 (1999).

${ }^{14}$ Y. Liang, W. D. Nix, P. B. Griffin, and J. D. Plummer, J. Appl. Phys. 97, 43519 (2005).

${ }^{15}$ F. Isa, M. Salvalaglio, Y. A. R. Dasilva, M. Meduňa, M. Barget, A. Jung, T. Kreiliger, G. Isella, R. Erni, F. Pezzoli, E. Bonera, P. Niedermann, P. Gröning, F. Montalenti, and H. von Känel, Adv. Mater. 28, 884 (2016).

${ }^{16}$ C. Chang, Y.-F. Wang, Y. Kanamori, J.-J. Shih, Y. Kawai, C.-K. Lee, K.-C. Wu, and M. Esashi, J. Micromech. Microeng. 15, 580 (2005).

${ }^{17}$ R. Bergamaschini, F. Isa, C. V. Falub, P. Niedermann, E. Müller, G. Isella, H. von Känel, and L. Miglio, Surf. Sci. Rep. 68, 390 (2013).

${ }^{18}$ C. Rosenblad, H. R. Deller, A. Dommann, T. Meyer, P. Schroeter, and H. von Känel, J. Vac. Sci. Technol. A 16, 2785 (1998).

${ }^{19}$ F. Isa, M. Salvalaglio, Y. A. R. Dasilva, A. Jung, G. Isella, R. Erni, P. Niedermann, P. Gröning, F. Montalenti, and H. von Känel, Acta Mater. 114, 97 (2016).

${ }^{20}$ V. A. Shah, A. Dobbie, M. Myronov, and D. R. Leadley, J. Appl. Phys. 107, 64304 (2010).

${ }^{21}$ S. Castellanos, M. Kivambe, J. Hofstetter, M. Rinio, B. Lai, and T. Buonassisi, J. Appl. Phys. 115, 183511 (2014).

${ }^{22}$ M. Salvalaglio and F. Montalenti, J. Appl. Phys. 116, 104306 (2014).

${ }^{23}$ F. Isa, A. Jung, M. Salvalaglio, Y. A. R. Dasilva, I. Marozau, M. Meduňa, M. Barget, A. Marzegalli, G. Isella, R. Erni, F. Pezzoli, E. Bonera, P. Niedermann, O. Sereda, P. Gröning, F. Montalenti, and H. von Känel, "Strain Engineering in Highly Mismatched SiGe/Si Heterostructures," Mater. Sci. Semicond. Process. (published online, 2016).

${ }^{24}$ A. K. Head, P. Humble, L. M. Clarebrough, A. J. Morton, and C. T. Forwood, Computed Electron Micrographs and Defect Identification (North-Holland Publishing Company, Amsterdam, 1973).

${ }^{25}$ K. Schwarz, Phys. Rev. Lett. 78, 4785 (1997).

${ }^{26}$ E. A. Fitzgerald, J. Vac. Sci. Technol. B 7, 782 (1989).

${ }^{27}$ F. Glas, Phys. Rev. B 74, 121302(R) (2006). 